Kerr, N. S. \& Sussman, M. (1958). J. gen. Microbiol. 19, 173-177

\title{
Clonal Development of the True Slime Mould, Didymium nigripes
}

\author{
By N. S. KERR AND M. SUSSMAN \\ Department of Biological Sciences, Northrestern University, Evanston, \\ Illinois, U.S.A.
}

SUMMARY: Didymium nigripes has been grown in two-membered cultures with Aerobacter aerogenes as its bacterial associate. All phases of the developmental cycle were observed, including spore germination, myxamoeboid growth, plasmodium formation and growth, and fruiting. Both spores and myxamoebae have regularly yielded clonal populations displaying normal development terminating with the appearance of fruits. Efficiency of plating determinations showed that spores and myxamoebae were $100 \%$ viable under the proper conditions. Greatly decreased spore viability was encountered when plasmodia were permitted to fruit in the presence of the metabolic products of their bacterial associate. Plasmodium formation was inhibited by the presence of $2 \%(w / v)$ glucose or $0.2 \%(w / v)$ brucine. However, the myxamoebae grew normally under these conditions.

The members of the Class Myxomycetes, Phylum Myxomycophyta, offer unique opportunities for the study of heterokaryosis and nuclear-cytoplasmic interrelationships. Unlike their relations, the cellular slime moulds (Acrasiales), they have been neglected as experimental organisms largely because of the difficulties encountered in finding strains which can be made to complete their life cycle reproducibly in the laboratory. The life cycle is as follows: the spores germinate liberating flagellate myxamoebae which undergo several divisions before fusing to form plasmodia. The plasmodia develop into large multinucleate masses which enter the stationary growth phase and differentiate into fruiting bodies. During this process meiosis occurs (Wilson \& Ross, 1955) and spores are formed. The conditions for spore germination, growth, and for the formation of fruiting bodies have unfortunately remained largely undefined. The complete life cycle has thus been only sporadically observed. It has been possible to surmount the various difficulties of cultivating these organisms in the case of Didymium nigripes. The present communication describes the cultural conditions which have permitted reproducible completion of the life cycle in less than 7 days with good spore viability and includes a method for the isolation and growth of clones.

\section{RESULTS}

Growth of mass cultures

Spores of Didymium nigripes were suspended by crushing fruiting bodies against the sides and bottom of a conical centrifuge tube which contained $1 \mathrm{ml}$. water. When the suspension was bubbled vigorously with air the stalks, clumps, and pieces of debris remained on the sides of the tube leaving a 
homogeneous spore suspension. A sample of this supension was spread together with a few drops of a $48 \mathrm{hr}$. Aerobacter aerogenes culture on SM/5 medium (2 g. Bacto-peptone; $2 \mathrm{~g}$. glucose; $0 \cdot 2 \mathrm{~g}$. yeast extract; $0 \cdot 2 \mathrm{~g}$. $\mathrm{K}_{2} \mathrm{HPO}_{4}$; $0 \cdot 3 \mathrm{~g} . \mathrm{KH}_{2} \mathrm{PO}_{4} ; 0 \cdot 2 \mathrm{~g}$. $\mathrm{MgSO}_{4} \cdot 7 \mathrm{H}_{2} \mathrm{O} ; 20 \mathrm{~g}$. agar; in 1 l. distilled water; final $\mathrm{pH}$ value $6 \cdot 0-6 \cdot 3)$. The plates were incubated at $22^{\circ}$. Spores germinated during the next $7 \mathrm{hr}$. to yield myxamoebae which soon developed flagella. Plasmodia appeared after 3 days, and continued to grow for $\mathbf{2}$ days until their bacterial food supply was exhausted. Fruiting bodies were then formed within $48 \mathrm{hr}$.

\section{Prolongation of the amoeboid stage}

The addition of $2 \%(\mathrm{w} / \mathrm{v})$ glucose or $0 \cdot 2(\mathrm{w} / \mathrm{v})$ brucine to the SM/5 medium inhibited plasmodium formation. Under these conditions spore germination and myxamoeboid growth took place as usual, but, instead of forming plasmodia, the amoebae continued to multiply until the plate was cleared of bacteria. When these amoebae were subcultured on similar media, they repeated this process. When subcultured to SM/5 medium they formed plasmodia which completed their life cycle normally. Sulphanilamide $(0.1 \%$, w/v) delayed plasmodium formation for $24 \mathrm{hr}$. when added to SM/5 medium but did not affect the growth of the myxamoebae.

\section{Isolation of clones}

Clones were obtained by using the method of Sussman (1951) originally devised to permit clonal growth of the cellular slime moulds, the Acrasiales. Samples containing up to fifty spores of myxamoebae were spread with Aerobacter aerogenes on SM/5 medium. Four days later small clear areas (plaques) containing myxamoebae appeared in the film of bacterial growth. These myxamoebae soon formed plasmodia which grew as a ring around the edge of the plaque and slowly enlarged it while feeding on the bacterial layer (Pl. 1, figs. 1-4). When the clones were about $1 \mathrm{~cm}$. in diameter they were transferred along with the underlying agar to $\mathrm{PA}$ medium $\left(0 \cdot 2 \mathrm{~g} . \mathrm{K}_{2} \mathrm{HPO}_{4} ; 0 \cdot 3 \mathrm{~g} . \mathrm{KH}_{2} \mathrm{PO}_{4}\right.$; $20 \mathrm{~g}$. agar; in 11 . distilled water; final $\mathrm{pH}$ value $6 \cdot 0-6 \cdot 3)$ and were allowed to fruit. Of eighteen clones thus tested, all fruited normally to yield viable spores which in turn produced clones which completed the life cycle in the usual manner. A single clone was selected for the remainder of the work reported here.

\section{Efficiency of plating}

A single empty spore case was often observed in a plaque (Pl. 1, figs. 2, 3) indicating that a single spore had given rise to the clone. It was necessary to determine, however, the proportion of spores which were viable and could produce normal plasmodia. This was accomplished by determining the ratio of spores plated to plaques formed (efficiency of plating, expressed as \%). Large plasmodia were transferred to PA medium and allowed to fruit. Two days later the fruits were picked, and the spores homogeneously suspended and counted in a Levy haemocytometer. The spore suspension was diluted appropriately and replicate samples containing up to fifty spores were spread, 
together with Aerobacter aerogenes, on SM/5 medium. Four days later clones appeared and were counted; the efficiency of plating of such spores approached $100 \%$. The degree of germination was also determined by observing microscopically the ratio of empty spore cases to total spores counted. Such observations yielded results which confirmed those obtained by the plating method. Spore viability remained near $100 \%$ for a month after fruiting. Myxamoebae when washed off plates of $2 \%(\mathrm{w} / \mathrm{v})$ glucose $+\mathrm{SM} / 5$ medium and plated in a similar manner, also had an efficiency of plating near $100 \%$.

When plasmodia were allowed to continue growing and to form fruiting bodies on the same plate which had supported their earlier growth, the viability of the resulting spores was exceedingly variable. The most likely cause of this irregularity seemed to be that metabolic products of the bacteria affected spore viability. This hypothesis was tested by scoring the viability of spores originating from plates which had been exposed to bacteria for varying periods of time. Sixteen plates of SM/5 medium were spread with Aerobacter aerogenes. Each day for 8 days two of these plates and a PA medium control plate were inoculated with plasmodia. Determinations of efficiency of plating were made on spores from each plate 2 days after the completion of fruiting. It can be seen from the results given in Table 1 that spore viability fell to zero after seven days of pre-incubation of the bacteria.

\section{Table 1. The effect of ageing of Aerobacter aerogenes on viability of spores} of Didymium nigripes

Spores in the experimental series were from plasmodia which had fruited on SM/5 medium after having been exposed to bacteria for varying periods of time. Control spores were from plasmodia which fruited on PA medium. EOP=efficiency of plating.

Experimental

\begin{tabular}{|c|c|c|c|c|c|c|}
\hline \multirow{2}{*}{$\begin{array}{l}\text { Days of } \\
\text { pre-incuba- } \\
\text { tion of } \\
\text { A. aerogenes }\end{array}$} & \multicolumn{2}{|c|}{1} & \multicolumn{2}{|c|}{2} & \multicolumn{2}{|c|}{ Control } \\
\hline & $\begin{array}{l}\text { No. of } \\
\text { plaques }\end{array}$ & $\begin{array}{l}\text { EOP } \\
(\%)\end{array}$ & $\begin{array}{l}\text { No. of } \\
\text { plaques }\end{array}$ & $\begin{array}{l}\text { EOP } \\
(\%)\end{array}$ & $\begin{array}{c}\text { No. of } \\
\text { plaques }\end{array}$ & $\begin{array}{c}\text { EOP } \\
(\%)\end{array}$ \\
\hline 0 & $38 \pm 8$ & 75 & $56 \pm 9$ & 112 & $45 \pm 3$ & 90 \\
\hline 1 & $32 \pm 2$ & 65 & - & - & $40 \pm 3$ & 80 \\
\hline 2 & $36 \pm 6$ & 72 & $52 \pm 3$ & 104 & 二 & - \\
\hline 3 & $22 \pm 7$ & 44 & $22 \pm 6$ & 44 & $49 \pm 2$ & 98 \\
\hline 4 & $12 \pm 8$ & 24 & $17 \pm 5$ & 34 & $53 \pm 11$ & 106 \\
\hline 5 & $23 \pm 2$ & 46 & - & - & $43 \pm 3$ & 86 \\
\hline 6 & $23 \pm 2$ & 46 & $6 \pm 5$ & 12 & $53 \pm 5$ & 106 \\
\hline 7 & 0 & 0 & $\overline{0}$ & 0 & $54 \pm 7$ & 108 \\
\hline
\end{tabular}

\section{DISCUSSION}

Brucine and glucose can conceivably act at either of two stages in the developmental cycle. These agents may either prevent the fusion of myxamoebae to form plasmodia, or, alternatively, having permitted fusion to occur, they may prevent the usual subsequent enlargement of the plasmodia. Against the latter possibility it should be noted that even newly formed plasmodia are readily distinguishable from myxamoebae on the basis of size, granulation and 
protoplasmic streaming. Thus it is reasonable to expect that they would have been detected on brucine or glucose plates had they been present. It would appear, therefore, that the first explanation can be tentatively accepted until such time as definitive studies with genetically marked stocks can be performed. The retention of the amoeboid stage by glucose or brucine has opened many interesting subjects to investigation because it is now possible to obtain myxamoebae in almost limitless quantities. Thus a quantitative analysis of the kinetics of plasmodium formation is practicable and is now being made. While it is difficult to measure accurately the growth of a plasmodium, it is easy to count an increase in the number of myxamoebae; their use should therefore simplify nutritional studies.

Spore viability. Cadman (1931) and Parker (1946) did not succeed in germinating single spores of Didymium; they postulated that interactions between spores were necessary for germination. There can be no doubt that isolated spores of our strain are capable of germination. When fifty spores are spread per plate the distance between plaques is often a centimetre. Interactions would not be likely to operate over this distance during the $7 \mathrm{hr}$. between plating and the end of germination. Because all spores are capable of developing singly our strain must be homothallic. The possibility that mating type is segregated during the first divisions after spore germination was eliminated when plasmodia and fruits were produced by clones derived from single amoebae.

The difficulty of obtaining viable spores is perhaps the principal factor which heretofore has discouraged laboratory cultivation of Myxomycetes. Although it is dangerous to generalize about a group as diverse as this one, the experiments reported here indicate that this difficulty may have been due to the use of spores which had been poisoned by metabolic products of the bacterial associate. Spores which regularly have a high degree of viability can be obtained in two ways. Either large plasmodia can be transferred to PA medium and be allowed to fruit, or, when large plasmodia are not available, plasmodia can be placed on SM/5 medium plates newly spread with bacteria. Because under the latter conditions the plates are inoculated with plasmodia instead of spores, fruiting occurs within 3 days rather than after 7 , and is therefore not affected by metabolic products of the bacterial associate (see Table 1).

We wish to thank Dr A. L. Cohen who kindly sent us the strain of Didymium nigripes used in this investigation.

This work was supported by research grants from the National Institutes of Health and the Office of Naval Research. A major portion of the experimental work reported is abstracted from a thesis written by the senior author (N.S.K.) in partial fulfilment of the requirements of the Ph.D. degree. A National Science Foundation pre-doctoral fellowship (1956-8) was held by one of us (N.S.K.). 

Journal of General Microbiology, Vol. 19, No. 1
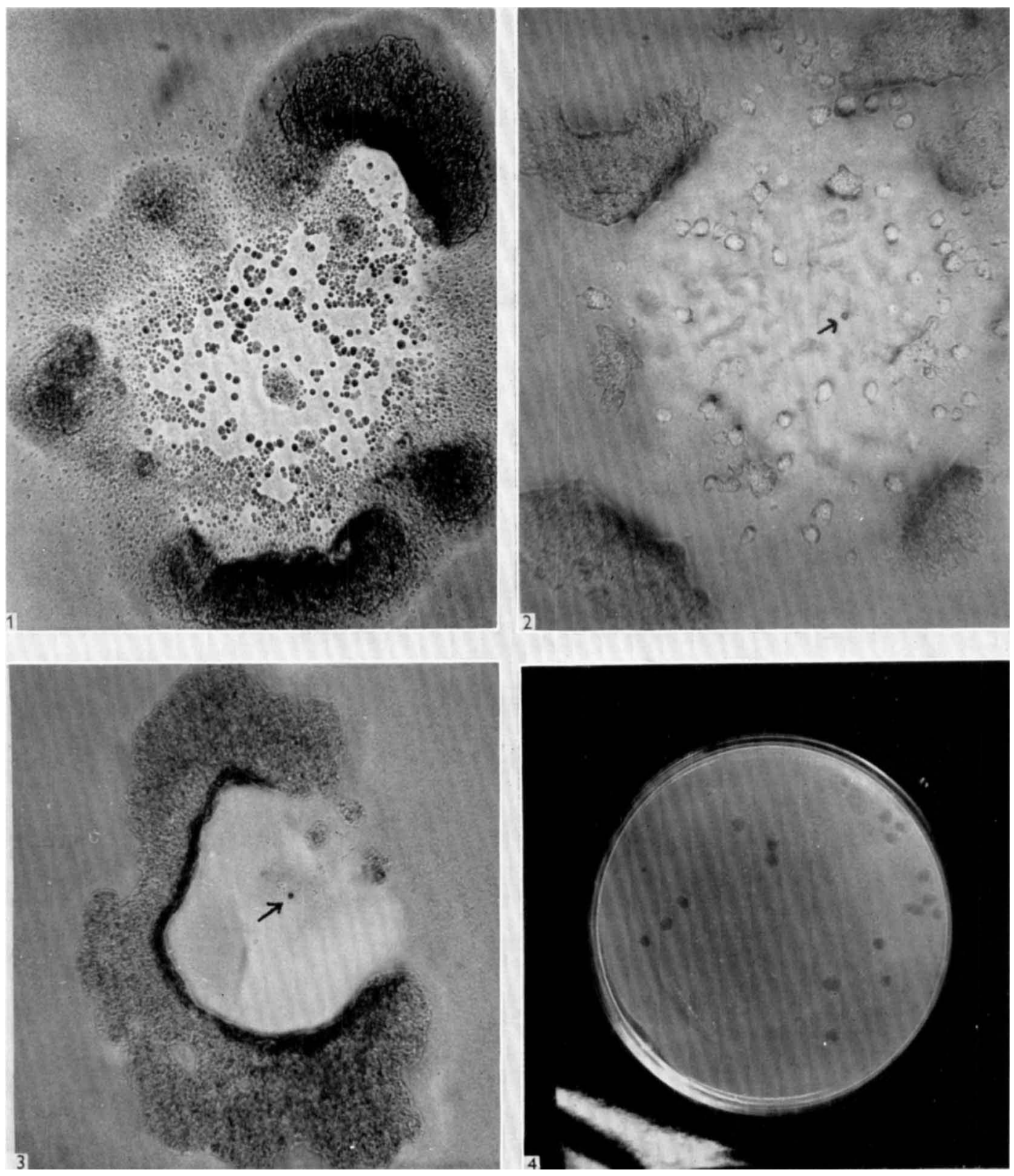

N. S. Kerr \& M. Sussman-Didymium nigripes. Plate 1 


\section{REFERENCES}

Cadman, E. J. (1931). The life history and cytology of Didymium nigripes. Trans. roy. Soc. Edinb. $57,93$.

Parker, H. (1946). Studies in the nutrition of some aquatic myxomycetes. $J$. Elisha Mitchell sci. Soc. 62, 231.

Sussman, M. (1951). Origin of cellular heterogeneity in the development of the slime molds, Dictyosteliaceae. J. exp. Zool. 118, 407.

Wirson, C. M. \& Ross, I. K. (1955). Meiosis in the myxomycetes. Amer. J. Bot. 42, 743.

\section{EXPLANATION OF PLATE}

Fig. 1. A young plaque. Myxamoebae, small plasmodia, and larger plasmodia are visible. $(\times 65$.

Fig. 2. A plaque showing small plasmodia and larger plasmodia. The spore case from which the clone arose is indicated by the arrow. $(\times 110$.

Fig. 3. A mature plaque. Most of the small plasmodia had fused into one large one which then fed on the bacteria at the edge of the plaque. The spore case from which the clone originated is indicated by the arrow. $(\times 65$.).

Fig. 4. A plate showing 19 clones. These plaques were slightly older than the one shown in figure 3. $\left(\times \frac{1}{3}.\right)$

(Received 7 February 1958) 